\title{
Tubérculos andinos y conocimiento agrícola local en comunidades rurales de Ecuador y Colombia
}

Neidy Lorena Clavijo Ponce*

Manuel Enrique Pérez Martínez**

Recibido: 2013-12-16 Aprobado: 2014-05-15 Disponible en línea: 2014-07-27

doi:I0.III44/javeriana.CRDII-74.taca

Cómo citar este artículo: Clavijo Ponce, N. y Pérez Martínez, M. (2014). Tubérculos andinos y conocimiento agrícola local en comunidades rurales de Ecuador y Colombia. Cuadernos de Desarrollo Rural, II(74), I49-166. http:// dx.doi.org/Io.III44/Javeriana.CRDII-74.taca

\section{Resumen}

En Ecuador y Colombia hay comunidades indígenas y campesinas que aún cultivan tres tubérculos andinos: Ullucus tuberosum, Oxalis tuberosa y Tropaelum tuberosum. Este documento, apoyado en el análisis etnoecológico del conocimiento agrícola local, explica mediante las prácticas de cultivo y creencias asociadas con estas especies, las determinantes para su conservación in situ en ambos países. Se muestra cómo las legislaciones vigentes, las instituciones, la migración y los usos del suelo, influyen de forma directa en sus áreas de siembra y técnicas de cultivo. Sin embargo, se destaca la persistencia de las creencias en torno a sus usos, vigente gracias a la memoria prevalente de la población adulta.

\section{Palabras clave:}

etnoecología; tubérculos andinos; conocimiento agrícola local; conservación in situ

\footnotetext{
* Ingeniera Agrónoma. Magister en Agricultura Ecológica. Estudiante de Doctorado en Agroecología. Docente-Investigadora, Facultad de Estudios Ambientales y Rurales. Línea de Investigación Agroecología e Innovación con comunidades rurales. Correo electrónico: n.clavijo@javeriana.edu.co ** Sociólogo. Magister en Planificación y Administración del Desarrollo Regional. Estudiante de Doctorado en Estudios Territoriales. Profesor Investigador. Pontificia Universidad Javeriana. Facultad de Estudios Ambientales y Rurales. Grupo de Investigación Institucionalidad y Desarrollo Rural.

Correo electrónico: manperez@javeriana.edu.co
}

\section{c) $\theta \otimes$}




\title{
Andean Tubers and Local Agricultural Knowledge in Rural Communities from Ecuador and Colombia
}

\begin{abstract}
In Ecuador and Colombia there are indigenous and peasant communities that still grow three Andean tubers: Ullucus tuberosum, Oxalis tuberosa and Tropaelum tuberosum. This paper, supported by a local ethnoecological analysis of the agricultural knowledge, explains the determinants for preservation in situ in both countries by means of cultural practices and beliefs associated with these species. It shows how existing laws, institutions, migration and land uses directly affect their planting areas and cultivation techniques. However, the persistence of beliefs about its uses stands out due to the prevalent memory of the adult population.
\end{abstract}

Keywords:

ethnoecology; Andean tubers; local agricultural knowledge; conservation in situ

\section{Tubercules andins et connaissance agricole local dans des communautés ruraux de l'Équateur et de Colombie}

\section{Résumé}

À l'Equateur et en Colombie il y a des communautés indigènes et paysans qui cultivent encore trois (3) tubercules andins : Ullucus tuberosum, Oxalis tuberosa et Tropaelum tuberosum. Ce document, fondé sur l'analyse ethnologique de la connaissance agricole locale, explique à travers des pratiques de culture et des croyances liées à ces espèces, les déterminants pour leur conservation in situ, dans les deux pays. Il montre comment les législations en vigueur, les institutions, la migration et l'utilisation du sol, influencent directement leurs champs ensemencés et leurs techniques de culture. Cependant, la persistance des croyances autour de leurs usages est encore mise en valeur grâce à la grande mémoire de la population adulte.

Mots-clés:

ethnologie; tubercules andins; connaissances agricoles locales; conservations in situ 


\section{Introducción}

En los Andes existen cultivos que son poco conocidos, incluso en los mismos países del área ya que suelen satisfacer demandas de carácter local (Ruíz, 2009). Entre estos se destacan tres especies en particular: La ruba (Ullucus tuberosum C), la ibia (Oxalis tuberosa M) y el cubio (Tropaeolum tuberosum RE॰P). Por lo general, están sembrados en pequeñas áreas bajo sistemas de producción tradicionales y en condiciones difíciles, pero son imprescindibles para asegurar la diversificación alimentaria y el sustento de las poblaciones que viven en mayor riesgo (Espinosa, Vaca, Abad y Crissman, 1997). Al parecer, la distribución de la variabilidad de estas especies no es uniforme en toda la franja andina; se concentra más bien en nichos denominados "microcentros" con características sociales, medioambientales y culturales favorables para la conservación natural de la biodiversidad (Cadima, 2006).

Uno de estos microcentros se encuentra en la región interandina del Ecuador, lugar de asentamiento de la comunidad indígena de las Huaconas, en la provincia de Chimborazo, un centro importante de conservación, cultivo y consumo de tubérculos andinos (Tapia, Estrella, Monteros, Valverde, Nieto y Córdova, 2004). Allí, el Instituto Nacional Autónomo de Investigaciones Agropecuarias del Ecuador (Iniap) reportó una gran riqueza etnobotánica en relación con diferentes nombres y usos que los agricultores les han asignado a estos cultivos (Barrera, Tapia y Monteros, 2004).

Por otra parte, en Colombia, mediante una investigación realizada por el Departamento de Desarrollo Rural y Regional de la Pontificia Universidad Javeriana, se identificó otro posible microcentro en los municipios de Turmequé y Ventaquemada en el departamento de Boyacá. Aquí, la presencia de rubas, ibias y cubios es evidente a diferentes escalas de siembra, desde su presencia en pequeñas franjas dentro de la huerta hasta en medianas áreas, que los convierten en cultivos secundarios de los sistemas productivos (Clavijo, 20II).

Tanto en la zona de las Huaconas en Ecuador como en Turmequé y Ventaquemada en Colombia se ha comprobado que los tres tubérculos andinos se mantienen en ámbitos en los cuales priman niveles de desarrollo económico muy bajos, pobreza, exclusión y marginación (Espinosa et al., 1997; Barrera et al., 2004; Clavijo, 20II). No obstante, estos cultivos están ligados íntimamente a su cultura campesina, razón por la cual sus expresiones culturales y técnicas se han mantenido desde la época precolombina hasta hoy.

En este orden de ideas, el objeto de este artículo es describir los contextos sociales, económicos y naturales, así como los marcos legales e institucionales en cada uno de los microcentros, con el fin de explicar las prácticas de manejo que los agricultores llevan 
a cabo en sus predios. Prácticas justificadas, además, por las creencias y valoraciones que implican en cuanto a la alimentación y usos medicinales. Estos elementos se presumen, inciden directamente en la conservación in situ de estas tres especies nativas.

\section{Precisiones conceptuales}

Al hablar de agrobiodiversidad se reconoce que esta ha sido creada a lo largo de miles de años por campesinas y campesinos mediante observación, selección, intercambio y mejoramiento, por tanto, estas personas desempeñan un papel central en su origen y mantenimiento (Casas y Parra, 2007). Con lo anterior se reconoce el protagonismo de las comunidades campesinas en los procesos de domesticación y conservación de especies vegetales, aspectos que denotan acciones individuales y colectivas orientadas a la conservación in situ. Esta última, se orienta a la participación activa de la familia campesina e involucra el mantenimiento de variedades tradicionales dentro de sistemas agrícolas locales (Tapia y Rosas, 1998). Además, la conservación in situ reconoce el conocimiento y las prácticas ancestrales de manejo de los agricultores como uno de los ejes fundamentales para su persistencia en el tiempo (Casas y Parra, 2007).

Tal conocimiento tiene un valor trascendente para clarificar las formas como los campesinos perciben y conceptualizan los recursos naturales de los que dependen para subsistir, más aún en una economía a pequeña escala. En esta concepción no se asume al campo cultivado como un ente aislado del resto de la realidad, sino como la representación de la forma que tienen los campesinos de relacionar el entorno, su cultura, su experiencia, la producción y la naturaleza, por medio de vivencias polivalentes y multidimensionales, en que las partes social y natural no tienen división (Morales, 2004). De esta manera, el conocimiento agrícola local puede concebirse a partir de un sistema complejo, compuesto de tres subsistemas: el contexto, la práctica y la creencia (Woodley, 2006).

* El conocimiento contextual representa el aprendizaje debido a la historia, los factores demográficos, las características biofísicas del lugar, así como los distintos actores que han sido portadores de nueva información (políticas de Estado, gobiernos locales, instituciones, organizaciones de base, etc.)

* El conocimiento como práctica representa la acción significativa mediante la interacción y el aprendizaje físico experimental. Es decir, todos los procesos que involucran la configuración y diseño de los sistemas productivos. 
* El conocimiento como creencia representa la influencia que la espiritualidad y los valores tienen sobre la manera como las personas actúan dentro de su ecosistema. De acuerdo con Woodley (2006), entender la complejidad del conocimiento de los agricultores sobre sus procesos de manejo, uso y conservación de recursos naturales, va más allá de consultar con la comunidad los nombres de especies, los sistemas de clasificación de plantas, usos locales, cambios climáticos, entre otros. Según el autor, estos procesos de consulta dirigida suelen extraer del contexto, el conocimiento de los agricultores de manera fragmentada, sin tomar en cuenta la interacción dinámica con una complejidad de variables. En este sentido, Follér (2002) propone considerar el conocimiento como parte de los procesos de la sociedad, con sus jerarquías de poder y con actores sociales diferentes que se oponen, reflejan, reproducen y también incorporan diferentes tipos de conocimiento.

\section{Metodología}

El método que se consideró para este estudio es el etnográfico, pues según Martínez (2004), es el de mayor preferencia para entrar a conocer un grupo étnico, racial o institucional que forma un todo particular y donde los conceptos de las realidades que se estudian adquieren significados especiales. La combinación del método etnográfico y el análisis del sistema complejo de conocimientos locales en relación con la conservación de agrobiodiversidad de tubérculos andinos, ubica esta investigación dentro del campo de la Etnoecología. Esta área del conocimiento multidisciplinario permite el estudio del conjunto de creencias (kosmos), el sistema de conocimientos (corpus) y el conjunto de prácticas productivas (praxis), con lo que se hace posible comprender el uso o manejo de la naturaleza y sus procesos (Toledo y Barrera, 2008).

La Etnoecología propone estudiar la integración del complejo kosmos-corpus-praxis dentro de los procesos de producción en las diversas escalas, así como comprender la realidad local mediante el estudio de las dinámicas sociales y de los factores naturales, en relación con un contexto particular. Por tanto, para cumplir con el objetivo de este estudio, treinta agricultores de cada microcentro', compartieron sus vivencias y experiencias sobre la conservación de tubérculos andinos por medio de instrumentos propios del método etnográfico: a) Entrevistas semiestructuradas,

I Los criterios de selección para los entrevistados se fundamentaron en dos aspectos: que vivan en las zonas y que sean cultivadores de tubérculos andinos con un rango de 15 a 20 años de tradición en sus prácticas. 
mediante las cuales se indagaron variables sociales, económicas, ecológicas, técnicas, así como las implicaciones de las diferentes entidades acompañantes en la zona; b) Observación participante, apoyada en recorridos por los dos microcentros, para así identificar prácticas, paisaje, presencia institucional y usos del suelo; c) Relatos de vida: quince agricultores por zona de estudio, reconstruyeron mediante su relato la experiencia de manejo, uso y conservación de tubérculos andinos y la relación que estos tienen con su familia y su comunidad.

\section{Resultados y discusión}

\section{I. El conocimiento agrícola local explicado desde el contexto}

La zona de las Huaconas se ubica en la región andina del Ecuador, provincia de Chimborazo, en un rango altitudinal entre los $2800 \mathrm{msnm}$ y los $3600 \mathrm{msnm}$. Perteneciente a la parroquia Cicalpa, en el cantón Colta ${ }^{2}$, forma parte de una de las regiones con mayor población indígena en el país (70\%). De acuerdo con Tapia, Estrella, Monteros, Valverde, Nieto y Córdova (2004) esta zona tiene una población rural aproximada de 4I2I habitantes: 2092 hombres y 2029 mujeres; incluye I8 comunidades, de las cuales San Isidro, La Merced, Coto Juan y Santa Isabel forman parte de este estudio.

Según el Plan de Ordenamiento Territorial de Colta (POTC, 20II), dentro de las actividades socioeconómicas más relevantes del cantón sobresale la agricultura, de hecho, $72.5 \%$ de la población económicamente activa se dedica a este rubro. Cultivos de habas (Vicia faba), cebada (Hordeum vulgare), papa (Solanum tuberosum), trigo (Triticum aestivum), quinua (Chenopodium quinoa), avena (Avena sativa) y otras hortalizas, constituyen su base productiva. Las rubas, cubios e ibias permanecen en áreas reducidas, generalmente para el consumo local. Sin embargo según testimonios de los agricultores, desde hace diez años atrás, la ganadería intensiva ha cobrado fuerza y ha desplazado en gran medida los anteriores sistemas productivos, situación que ha originado importantes cambios en la configuración de su paisaje.

De otra parte, de acuerdo con Lima y Logroño (2005), el crecimiento demográfico y la escasez de tierra en el cantón han dado paso a uno de los fenómenos que han afectado más a los procesos productivos en la zona: la migración. Cerca de 27\% de la población de la provincia de Chimborazo emigra hacia las grandes ciudades del país, y cerca de

2 Los cantones de Ecuador son las divisiones administrativas de segundo nivel. La República del Ecuador está dividida en 24 provincias que a su vez están divididas en 22I cantones. Los cantones a su vez están subdivididos en parroquias, las que se clasifican entre urbanas y rurales. 
I.8\% de la población busca futuro en el exterior. Este factor ha propiciado cambios en el uso del suelo, ahora enfocado en la ganadería extensiva y cultivo de pastos, lo que está afectando la preservación y conservación de tubérculos andinos, sustituidos de forma notable por alimentos procesados bajo estándares de consumo comercial.

Como en el resto de la provincia de Chimborazo, gran parte de la población en el cantón Colta se encuentra ubicada en zonas relacionadas con los páramos, donde la situación de pobreza es crítica (Beltrán, Bustamante, Cuesta, De Biévre, Albán y Arguello, 20II). De hecho, Colta, se encuentra entre los diez cantones más pobres del país. Para Chiriboga y Wallis (2010) esta pobreza se ve reflejada en necesidades básicas insatisfechas y desnutrición crónica infantil, aspectos en los que Colta sobresale a escala nacional.

Por otro lado, en Colombia, el municipio de Turmequé se encuentra en el departamento de Boyacá, pertenece a la provincia de Márquez, y está situado a una altura de $2389 \mathrm{msnm}$, con temperatura promedio de $\mathrm{I} 4{ }^{\circ} \mathrm{C}$. Se caracteriza por depender económicamente del sector agrícola y de actividades artesanales, como los tejidos y la elaboración de amasijos. Abastece gran parte del mercado local de alimentos y aporta en la provisión de algunos mercados regionales (EOTT, 1999). Próximo a Turmequé se encuentra el municipio de Ventaquemada, ubicado a una altitud de $2630 \mathrm{msnm}$. Su principal factor de desarrollo son las actividades agropecuarias, cuya característica es la ocupación de mano de obra familiar. Ventaquemada tiene como producto base la papa, con aproximadamente 5000 ha, con una producción cercana a 84000 toneladas.

Otros cultivos como la alverja y los frutales tienen gran importancia en la economía campesina y en la canasta familiar. En ambos municipios la estructura de la tierra se caracteriza por la existencia de microfundios, donde la mayoría de los predios tiene un área mayor a una ha y menor de tres ha. En menor proporción se cuentan los microfundios, predios mayores de 5 ha y menores de io ha (IGAC, 2012). Dentro de los cultivos anuales se destaca el maíz (Zea mays), que se siembra asociado con fríjol (Phaseolus vulgaris), haba (Vicia faba) o arveja (Pisum sativum). Frutas como mora (Rubus glaucus Benth), uchuva (Physalis peruviana L.), curuba (Passiflora tripartita), papayuela (Vasconcellea cundinamarcensis), tomate de árbol (Solanum betaceum), ciruelo (Prunus domestica) y durazno (Prunus persica). Ibias, cubios y rubas, generalmente se cultivan para autoconsumo.

$\mathrm{Al}$ igual que en el Ecuador, el uso tradicional del suelo con vocación agrícola se ha visto paulatinamente cubierto por la ganadería extensiva, con predominio de especies de doble propósito: Holstein y Brown Swiss. Ocupación que, en gran 
medida, ha sido acogida por los adultos y adultos mayores. De otra parte, Turmequé y Ventaquemada, reportan necesidades básicas insatisfechas, con tendencia a incrementarse en las zonas rurales, donde alcanzan 39.93\% y 29.44\% (DANE, 2005). Al igual que en las Huaconas, según prospecciones del DANE, su población rural tiende a la disminución, debido a los procesos migratorios de jóvenes y adultos, en su mayoría a la capital departamental y Bogotá. Producto de ello, la escasez de mano de obra para el manejo y conservación de las especies cultivadas no se ha hecho esperar.

En cuanto al contexto legislativo, tanto Ecuador como Colombia señalan en su Constitución la importancia que representa para sus Estados la producción de alimentos y la protección de los recursos naturales asociados. De igual forma respaldan la generación de nuevas tecnologías y la producción de conocimiento alrededor de los cultivos. No obstante, la carta magna ecuatoriana, reformada hace cinco años bajo la concepción del Buen Vivir, acoge como uno de sus argumentos centrales el fortalecimiento de su Soberanía Alimentaria ${ }^{3}$. Esto implica la protección del mercado doméstico contra los productos excedentarios que se venden más baratos en el mercado internacional, así como la valoración del conocimiento local de los pueblos indígenas (Proaño, 20II).

En Colombia, el escenario es distinto. El desarrollo del sistema productivo agropecuario obedece a las orientaciones del modelo de competitividad a partir de la industrialización del campo. En este sentido, el fin es responder a las demandas comerciales internacionales, en razón de los tratados de libre comercio firmados recientemente, así como a las demandas nacionales de alimentos y materias primas. Sin embargo, lo anterior no excluye la coexistencia de modelos alternativos de producción fundamentados en principios de diversificación y manejo sostenible (Corrales y Forero, 2007; León, 2010; PNUD, 20II; Pérez y Clavijo, 20I2; Clavijo, 20I3).

Planteado de esta manera, parecería que en Ecuador, para el caso particular de los tres tubérculos andinos, el panorama es favorecedor, pues existen leyes que propician el cultivo, comercialización y manejo de alimentos cuya tradición va más allá del factor alimentario. No obstante, si bien su conservación podría obedecer a factores de orden legal, tales elementos no son determinantes, pues la ejecución de los mandatos expuestos en la Constitución se hacen efectivos por medio del Ministerio de Agricultura, Ganadería, Acuicultura y Pesca (Magap) y el Gobierno Autónomo Descentralizado de la Provincia (GADP).

3 Se entiende como la facultad de cada Estado para definir sus propias políticas agrarias y alimentarias de acuerdo a objetivos de desarrollo sostenible y seguridad alimentaria. Ello implica la protección del mercado doméstico contra los productos excedentarios que se venden más baratos en el mercado internacional, y contra la práctica del dumping (Delgado y Escobar, 2009). 
Es así como, desde la dirección de este Ministerio en la provincia de Chimborazo, se reconoce y valora la importancia de los tubérculos andinos, aunque se asume que su producción y conservación no han sido priorizadas, situación que también la comparte el GADP de Chimborazo. Esto coincide con las respuestas dadas por los agricultores entrevistados en el sector de las Huaconas, al señalar que durante los últimos diez años ${ }^{4}$ no han recibido apoyo estatal para la conservación y uso de los tres tubérculos. En su defecto, las instituciones presentes en la zona promueven la ganadería extensiva.

En Colombia el panorama no difiere. El Ministerio de Agricultura y Desarrollo Rural (MADR) no prioriza dentro de sus cadenas productivas los cultivos objeto de este estudio, ni tampoco destina recursos a proyectos de investigación y procesos de capacitación sobre estas especies. Actualmente, las directrices de esta entidad promueven de manera notoria el encadenamiento productivo, la generación de valor agregado y el fortalecimiento empresarial de cultivos más comerciales.

Desde la Secretaria de Fomento Agrícola del Departamento de Boyacá, ente territorial que coordina y ejecuta lo establecido desde el MADR, el cultivo y conservación de cultivos de ruba, ibia y cubio no son una prioridad. Esto se debe a la noción de que son productos con una incipiente demanda en el mercado, lo cual implicaría bajos ingresos para el agricultor por este rubro. En este escenario, se podría pensar que organismos no gubernamentales en los dos países podrían haber ocupado el lugar del Estado y adelantar actividades relacionadas con la conservación de estos tubérculos. Sin embargo, la apuesta también ha sido reducida.

Según los habitantes de la zona, a excepción de algunos talleres de capacitación sobre seguridad alimentaria que en 20 Ir brindó la FAO en las Huaconas, en los cuales se fomentaba el uso de cultivos andinos en la elaboración de recetas, ninguna otra entidad externa al Estado ha estimulado el uso y conservación de estas especies. Mientras, en Turmequé y Ventaquemada, solo hasta el año 2008 la Pontificia Universidad Javeriana, junto con la Corporación para el Desarrollo Participativo y Sostenible de los Pequeños Agricultores Colombianos (Corporación PBA), adelantaron procesos de investigación y fomento de conservación de ibias, cubios y rubas, en pequeños sistemas productivos (Clavijo, Combariza y Barón, 20I2).

Este escenario, explicado desde aspectos socioecológicos e institucionales, da la pauta para determinar que el proceso sistemático de la pérdida de diversidad

\footnotetext{
4 Después del trabajo llevado a cabo por el Instituto Nacional de Investigaciones Agropecuarias en colaboración con el Centro Internacional de la Papa y Condesan, los pobladores aducen que nada se ha conservado por falta de seguimiento.
} 
de tubérculos andinos, así como la reducción de sus áreas de cultivo, disminución de sus usos y consumo en las dos zonas de estudio, no son hechos aislados, sino que corresponden a los efectos propiciados por componentes externos a la comunidad, los cuales están definiendo sus mensajes y acciones.

\subsection{El conocimiento agrícola local explicado desde las prácticas}

Los agricultores de las Huaconas, Turmequé y Ventaquemada coinciden en que a partir de los años setenta, la difusión de prácticas relacionadas con la agricultura industrializada intensificó la mecanización agrícola, el uso de productos químicos y la modalidad de monocultivos. Prácticas difundidas desde los Ministerios de Agricultura y casas comerciales de ambos países, que orientaron la modernización de los procesos productivos para incrementar la productividad y por tanto sus excedentes comerciales. De este modo, los pequeños agricultores se volvieron viables para el mercado, en respuesta al modelo de desarrollo neoclásico predominante en la época (Mora, 2008; Leff, 2008). Como consecuencia, la disminución en el cultivo y consiguiente conservación de especies locales no se hicieron esperar (Pengue, 2005).

El incremento de áreas sembradas con cultivos comerciales se ha establecido en detrimento de aquellas destinadas a la alimentación diversificada de sus pobladores, en particular, de las tres especies objeto de este estudio. Frente al incremento de la producción ganadera en estas zonas, los campesinos e indígenas de Ecuador y Colombia aseveran que esto obedece a que el cuidado del ganado resulta menos riesgoso y laborioso que los cultivos. En efecto, los cultivos requieren prácticas de control fitosanitario y mano de obra familiar, recurso que en el campo ha disminuido debido a los procesos migratorios de sus pobladores más jóvenes.

De esta manera, los adultos y adultos mayores que permanecen en las zonas rurales se ocupan de dos o tres cabezas de ganado, de las cuales obtienen, por lo menos, el ingreso diario de la venta de leche, equivalente a cinco dólares en promedio. Lo anterior con prácticas básicas de ordeño, pastoreo y, cuando la ocasión lo amerita, de cuidados sanitarios. Paradójicamente, la prevalencia de estas poblaciones influye directamente en la persistencia de tubérculos andinos y otras especies nativas, y junto con estas, varias de sus prácticas tradicionales de cultivo.

Precisamente, son los abuelos, padres y madres de las actuales generaciones quienes valoran, reconocen y recuerdan su diversidad, sus usos, sabores y formas de manejo. Esto ratifica la postura de Tapia (2002, p. 79) al afirmar que "es común que los miembros más viejos de una comunidad posean conocimientos mejores y más detallados que los jóvenes”; según el autor, los jóvenes están imbuidos de la cultura 
moderna alienante, por cuanto este tipo de conocimientos se va perdiendo cada vez con más rapidez. Así lo señala una agricultora de Ventaquemada: “Los jóvenes de hoy en día no echan azadón, por eso es que se está terminando todo, al menos si se van a la ciudad se les olvida esta comida. Por eso, son los mismos ancianos que siembran estos cultivos, solo para comer”.

En las Huaconas, los pobladores indígenas adultos y adultos mayores, reconocen estos tubérculos como cultivos antiguos o comida de sus abuelos. Los catalogan como cultivos limpios porque no requieren de productos químicos y los asumen como fuente de salud y alimento. Históricamente, para ellos son cultivos que se siembran después de la papa y en asocio con otras especies, por lo general, habas, aunque hace años se acostumbraba rotar con cebada y quinua, ya ausentes en la localidad. Dada la altitud de la zona, los tres tubérculos solo tienen una cosecha anual y sus épocas de siembra se concentran en los meses de septiembre a diciembre. En cuanto a las variedades por especie, los agricultores hacen referencia a las que aún predominan en sus campos (Tabla I), pese a que en el pasado se destacaban morfotipos de colores diversos, hoy ausentes en la zona (Tapia et al., 2004).

Tabla l. Conocimientos de agricultores de las Huaconas sobre el cultivo y características de tres tubérculos andinos. 2013

\begin{tabular}{|c|c|c|}
\hline $\begin{array}{c}\text { ULLUCUS TUBEROSUM } \\
\text { RUBA-MELLOCO } \\
\end{array}$ & $\begin{array}{c}\text { OXALIS TUBEROSA } \\
\text { IBIA-OCA }\end{array}$ & $\begin{array}{c}\text { Tropaelum tubERosum } \\
\text { Cubio-MASHWa }\end{array}$ \\
\hline $\begin{array}{l}\text { Es considerado el cultivo } \\
\text { con mejor valoración en } \\
\text { precio y distribución en } \\
\text { los mercados locales. }\end{array}$ & $\begin{array}{l}\text { Generalmente se consume } \\
\text { en familia. Tiene incipiente } \\
\text { margen de negociación } \\
\text { en el mercado. }\end{array}$ & $\begin{array}{l}\text { Es un cultivo que ya no } \\
\text { existe en la zona. Dejó de ser } \\
\text { consumido aproximadamente } \\
\text { hace diez años, debido a las } \\
\text { barreras comerciales y de la } \\
\text { pérdida de conocimiento sobre } \\
\text { sus usos y beneficios por parte } \\
\text { de las nuevas generaciones } \\
\text { de pobladores en la zona. }\end{array}$ \\
\hline $\begin{array}{l}\text { Se conservan cuatro variedades: } \\
\text { caramelo, gallo, ambateño } \\
\text { blanco y cuencano. De } \\
\text { estas, las tres primeras son } \\
\text { las que más se cultivan. }\end{array}$ & $\begin{array}{l}\text { Se conocen tres tipos: blancas } \\
\text { (más apetecidas), amarillas y } \\
\text { negras (ya casi no existen). }\end{array}$ & $\begin{array}{l}\text { No recuerdan sus } \\
\text { características y variedades. }\end{array}$ \\
\hline Ciclo: 6-7 meses & Ciclo: $7-9$ meses & Ciclo: 7-9 meses \\
\hline \multicolumn{3}{|c|}{ Época de siembra: septiembre-diciembre; se cosechan una vez al año } \\
\hline $\begin{array}{l}\text { Suelen sembrarse con habas y pa } \\
\text { aunque a veces la ruba se siembr }\end{array}$ & & \\
\hline
\end{tabular}


Por otra parte, la disponibilidad de la semilla es proporcional a la disminución de áreas de cultivo y diversidad de morfotipos. Frente a esto, los pobladores afirman que cada vez es más difícil conseguir las semillas mediante venta e intercambio entre vecinos, como solía ocurrir. Ahora, la manera más común de obtenerlas es comprarlas en mercados locales.

En Turmequé y Ventaquemada los adultos y adultos mayores también perciben los tres tubérculos andinos de manera similar a sus vecinos ecuatorianos, es decir, como cultivos sanos, costumbre de sus ancestros, fuente de alimentación y vida. A diferencia de lo que ocurre en las Huaconas, los cubios se consumen y cultivan en mayor proporción que la ruba y las ibias. Según las tradiciones indígenas de la zona, aún se siembran en asociación con otros cultivos, como habas, maíz, arracacha, cucurbitáceas y arveja; o después de un cultivo comercial, como papas o fríjol, para que puedan usar los remanentes de fertilización en el suelo.

Las áreas destinadas a su cultivo también son reducidas, si se considera, como ya se ha dicho, que los sistemas productivos donde se cultivan corresponden a mini y microfundios. Tanto la ruba como las ibias tienen una sola cosecha al año, mientras que los cubios podrían cubrir hasta dos ciclos anuales, sobre todo en las partes bajas de los dos municipios, es decir, sobre los $2450 \mathrm{msnm}$. Las fechas de siembra inician en octubre y pueden extenderse hasta enero, aunque el cubio puede sembrarse también en mayo y junio. Los instrumentos de siembra tradicionales son el azadón y en algunas fincas todavía el arado con bueyes (Tabla 2).

TABla 2. Conocimientos de agricultores de Turmequé y Ventaquemada sobre el cultivo y características de tres tubérculos andinos. 2013

\begin{tabular}{|c|c|c|}
\hline $\begin{array}{c}\text { ULLUCUS TUBEROSUM } \\
\text { Ruba-MELLOCo }\end{array}$ & $\begin{array}{l}\text { OXALIS TUBEROSA } \\
\text { IBIA-OCA } \\
\end{array}$ & $\begin{array}{c}\text { TROPAELUM TUBEROSUM } \\
\text { CUBio- MASHWA }\end{array}$ \\
\hline $\begin{array}{l}\text { Ocupa el segundo lugar de } \\
\text { importancia entre los tres. } \\
\text { Su mucílago" no les gusta } \\
\text { a los niños y jóvenes. }\end{array}$ & $\begin{array}{l}\text { Hoy se consume muy poco en } \\
\text { familia. No tiene mucha salida } \\
\text { en el mercado. Es el tubérculo } \\
\text { que menos se consume y vende. }\end{array}$ & $\begin{array}{l}\text { De los tres, es el que mejor } \\
\text { aceptación tiene en el } \\
\text { mercado. Su sabor dulce lo } \\
\text { hace atractivo entre los niños }\end{array}$ \\
\hline $\begin{array}{l}\text { Se conservan cuatro tipos de rubas: } \\
\text { rojas, amarillas, rosadas y verdes. }\end{array}$ & $\begin{array}{l}\text { Se conocen las ibias rosadas, } \\
\text { amarillas, blancas y } \\
\text { morfotipos rayados (blancas y } \\
\text { amarillas con rayas rojas). }\end{array}$ & $\begin{array}{l}\text { Amarillos, blancos, rojos o } \\
\text { morados y morfotipos rayados } \\
\text { (blancos y amarillos con rayas } \\
\text { negras, moradas y rojas). }\end{array}$ \\
\hline Ciclo: 5-6 meses & Ciclo: 7-9 meses & Ciclo: 7-9 meses \\
\hline \multicolumn{3}{|c|}{ Época de siembra: septiembre-diciembre; se cosechan una vez al año. } \\
\hline \multicolumn{3}{|c|}{ Generalmente se siembran en asociación con otros cultivos, o después de un cultivo grande como papas o fríjol. } \\
\hline \multicolumn{3}{|l|}{ Fuente: elaboración propia } \\
\hline * Tipo de fibra soluble de característica visc & & \\
\hline
\end{tabular}


También se señala que la semilla se obtiene por tres vías: la primera, de su propia producción; la segunda, por la compra y venta entre vecinos; y la tercera, según el flujo de precios, se adquiere en las tiendas o en el mercado local. Buena parte de la cosecha se destina para alimentación familiar, aunque debido a la deficiencia en procesos de almacenamiento suelen tener pérdidas equivalentes a $40 \%$ de la cosecha. Para ellos, los tubérculos andinos marcan la diferencia pues se enorgullecen de su producción, basada en abonos orgánicos provenientes de residuos de cosecha y deyecciones animales, además de la nula aplicación de productos químicos: aducen que son resistentes a plagas y enfermedades debido a su rusticidad, pues son especies adaptadas desde hace siglos a la zona.

\subsection{El conocimiento agrícola local explicado desde las creencias}

Según Woodley (2006), la comprensión de la base epistemológica del conocimiento de los agricultores está ligado más al por qué, que al cómo. En este caso, acercarnos a las razones por las cuales las comunidades campesinas e indígenas de las dos zonas de estudio, a pesar de cada contexto, aún cultivan, así sea en pequeñas cantidades, tubérculos andinos, corresponde a una primera aproximación al entendimiento de ese por qué. En este sentido, se pudo determinar que la justificación para el cultivo de la ibia, el cubio y la ruba, se fundamenta en dos grandes pilares: el conocimiento sobre sus aportes alimenticios y la influencia de estos en la salud, dada las propiedades medicinales que les adjudican.

Sobre lo primero, en ambos microcentros se considera que el consumo de estas especies es un importante aporte a la dieta, debido a que se producen en sus campos, les otorga fuerza, resistencia y los ha mantenido saludables a lo largo del tiempo. $\mathrm{Al}$ respecto, las personas hacen remembranza del vigor que sus abuelos hasta ahora manifiestan, cualidad que le atribuyen al consumo constante, no solo de los tres cultivos sino a su combinación con otras especies andinas. Tanto el cocido boyacense en Colombia, como el timbo y el cariucho en Ecuador', preparaciones muy similares, representan lo anterior, tal como lo señala un agricultor de las Huaconas: "Esta comida nos mantenía fuertes, yo recuerdo cuando entraba a la escuela, mis padres

5 Cocido boyacense: plato tradicional de la región que incluye además de estos tubérculos, ingredientes como habas, arvejas, papas, carne de cerdo y de res. Timbo: un cocido de habas, papas, ibias y cebolla que condimentado solo con un poco de sal, lo llevaban de fiambre. Cariucho: Una mezcla se choclo o maíz tierno, papas cocidas, rubas, ibias, cubios y habas, que se come acompañado de queso o ají. 
me mandaban para el almuerzo el timbo, haciendo cucayo ${ }^{6}$ porque las clases eran de 7 a 4 pm. Los domingos se hacía cariucho y comíamos entre todos”.

Además de lo enunciado, otros platos con distintas combinaciones simbolizan la versatilidad en el consumo de los tubérculos andinos, como una expresión del arraigo que han tenido dentro de las dos comunidades; preparaciones que las mujeres de los dos microcentros detallaron para este trabajo. Investigaciones llevadas a cabo previamente en ambas zonas (Espinosa et al., 1997; Barón, Clavijo y Combariza, 20I0), recopilaron e innovaron otras recetas que, al parecer, toman como base las formas tradicionales de consumo, inventariadas en esta investigación (ver Tabla 3).

Tabla 3. Preparaciones tradicionales con tubérculos andinos en la Huaconas, Turmequé y Ventaquemada (2013)

\begin{tabular}{cc}
\hline Las Huaconas, provincia de & Turmequé y Ventaquemada, \\
Chimborazo, Ecuador & departamento de Boyacá, Colombia \\
\hline
\end{tabular}

Locros (sopas espesas combinadas con papa) Cocinados con guiso con cebolla y tomate

\begin{tabular}{ll}
\hline $\begin{array}{l}\text { Menestra (una cocción con sal, } \\
\text { leche, y condimentos) }\end{array}$ & Coladas, chicha, masato y mermeladas \\
\hline $\begin{array}{l}\text { Jugo de rubas (una vez cocidas, se licúan en } \\
\text { la misma agua y se endulza con panela) }\end{array}$ & $\begin{array}{l}\text { Se cocinan y se sirven como complementos } \\
\text { de piquetes y sancochos }\end{array}$ \\
\hline Coladas dulces y coladas saladas (cremas) & $\begin{array}{l}\text { Sopa de ibias y las rubas, con } \\
\text { pellejo de cerdo picado }\end{array}$ \\
\hline $\begin{array}{l}\text { Ensaladas en mezcla con cebolla roja, tomate } \\
\text { y cilantro. Principalmente con rubas cocidas }\end{array}$ & Ají de rubas (cebolla, tomate, ají y calabaza) \\
\hline Chicha de cubios & $\begin{array}{l}\text { Ibias combinadas con habas o } \\
\text { con apio de arracacha }\end{array}$ \\
\hline Dulce de cubios e ibias, con panela y canela & Sopa de mute con ibias
\end{tabular}

Fuente: elaboración propia

Adicionalmente, en ambos microcentros existen creencias vinculadas con sus propiedades medicinales: durante años han sido utilizadas en las dos comunidades como elementos curativos o preventivos para enfermedades renales, circulatorias y digestivas. $\mathrm{Al}$ respecto, se le atribuyen los siguientes efectos: mejoran la digestión,

6 Similar a una lonchera, tiene relación con los alimentos que los antepasados llevaban para soportar largos trayectos. 
alivian el dolor de huesos, mejoran la circulación sanguínea, limpian el hígado, limpian canales urinarios, desinflaman la próstata, contrarrestan infecciones del riñón, combaten la gastritis, aminoran el origen y los efectos del reumatismo, disminuyen los problemas de hipertensión arterial, limpian la piel, son cicatrizantes y antiinflamatorios. En cuanto a prácticas de uso terapéutico se destacan:

* Las rubas: se cortan en tajadas muy delgadas y se ponen crudas en la piel para que cicatrice.

* Los cubios: son valorados como un producto capaz de contrarrestar el incremento de ácido úrico en la sangre. Ingerir el cubio en agua tibia puede tener efectos curativos.

* Los morfotipos de cubios de color morado: afirman que consumirlos en infusión puede curar la hipertensión y la diabetes.

* Las rubas licuadas: si se toma su extracto en ayunas por nueve días, combate enfermedades de insuficiencia renal.

* Como purgante: "Se toman 3 cubios pequeños, se licúan, se consumen en ayunas, tipo 4 de la mañana y se espera hasta las 4 de la tarde para una segunda toma. Así, saca los cálculos del hígado, de los riñones y hace una limpieza al hígado y a todo el organismo" (Agricultor de Turmequé).

Mientras se les reconozca su utilidad y exista memoria sobre ellas, la probabilidad de su conservación es alentadora, ya que además del uso básico en alimentación, en ambos microcentros los agricultores coinciden en atribuirles valores medicinales, económicos y culturales.

\section{Conclusiones}

En referencia a las prácticas y creencias en el uso y conservación de los tubérculos andinos es evidente que estos conocimientos están seriamente amenazados por los contextos institucionales, que no salvaguardan de forma efectiva y directa la protección de estas prácticas de cultivo. En suma, las orientaciones de los modelos de desarrollo agrícola para América Latina demuestran la tendencia de un modelo global de desarrollo, que otorga limitados espacios en el mercado a la agricultura tradicional, por lo general, ya marginada. Lo anterior profundiza la tendencia a la desaparición de estas especies, así como de los sistemas de producción que las mantienen. Aunado a esto, las implicaciones del cambio cultural que incide en las 
relaciones intergeneracionales en el medio rural, como lo demuestra este estudio, incrementan la dificultad de mantener las prácticas y valoraciones asociadas, y de lograr un reconocimiento por parte de las políticas y programas públicos de seguridad y soberanía alimentaria.

Esta circunstancia es una evidencia de lo expuesto por Woodley (2006) y Follér (2002), quienes consideran la complejidad del conocimiento de los agricultores como un resultado de factores internos y externos que configuran su dinamismo. Así, el conocimiento de las nuevas generaciones se está configurando con base en escenarios locales dados por otros usos del suelo, patrones de consumo y demandas externas. En este sentido, el conocimiento agrícola local deja de vincularse únicamente con los procesos históricos y tradicionales, pues también se nutre de las innovaciones que llegan a la zona, las cuales, en este caso, no contribuyen en lo absoluto a la conservación de tubérculos andinos.

No obstante, se espera que estas experiencias propicien la posibilidad de aumentar en centros de investigación y en la academia estudios sobre el conocimiento agrícola local, en especial, en los ámbitos de medicina, encadenamiento productivo alternativo o protección de derechos a los cultivadores. A su vez, se busca despertar el interés para la realización de pesquisas en los géneros y especies que los agricultores han preservado in situ, así como su mejoramiento genético. Se pretende también, y a partir de cada contexto, indagar sobre las prácticas y las creencias basadas en las costumbres y tradiciones culturales.

\section{Referencias}

Barón, M., Clavijo, N. y Combariza, J. (2010). Recetario. Tubérculos andinos de Turmequé $y$ Ventaquemada. Bogotá D. C.: Javegraf.

Barrera, V., Tapia, C. y Monteros, A. (2004). Raíces y tubérculos andinos. Alternativas para la conservación y uso sostenible en el Ecuador. Quito: Iniap.

Beltrán, K., Bustamante, F., Cuesta, B., De Biévre, M., Albán, M. y Arguello, M. (20II). Los páramos de Chimborazo. Un estudio socioambiental para la toma de decisiones. Quito: Condesan.

Cadima, X. (2006). Tubérculos. En R. Moraes, B. Øllgaard, L. Kvist, F. Borchsenius \& H. Balslev (Eds.), Botánica Económica de los Andes Centrales. Universidad Mayor de San Andrés. La Paz: Plural Editores. 
Casas, A. y Parra, F. (2007). Agrobiodiversidad, parientes silvestres y cultura. Leisa, Revista de Agroecología, 23(2), I-8.

Chiriboga, M. y Wallis, B. (2010). Diagnóstico de la pobreza rural en Ecuador y respuestas de la política pública. Quito: Rimisp.

Clavijo, N. (20II, abril). Tubérculos andinos en Boyacá. Un caso de innovación participativa. En Pontificia Universidad Javeriana (Comp.), VII Seminario de Desarrollo Rural. Mundos rurales y transformaciones globales: desafios y estrategias de respuesta (pp. 62-7I). Bogotá D. C.: Editorial Pontificia Universidad Javeriana.

Clavijo, N., Combariza, J. y Barón, M. (2012). Recognizing Rural Territorial Heritage: Characterization of Andean Tuber Production Systems in Boyacá. Revista Agronomía Colombiana, 29(2), 315-322.

Clavijo, N. (2013). Agroecología en los Andes. Bogotá D. C.: Editorial Pontificia Universidad Javeriana.

Corrales, E. y Forero, J. (2007). La reconstrucción de los sistemas de producción campesinos. El caso de Asproinca en Rio Sucio y Supía. Bogotá D. C.: Swissaid.

Delgado, R. y Escobar, C. (2009). Innovación tecnológica, soberanía y seguridad alimentaria. La Paz: Agruco.

DANE (2005). Proyecciones municipales de población 2005-20II. Sexos y grupos de edad. Recuperado el I2 de octubre 20I3, de http://www.dane.gov.co/files/investigaciones/ poblacion/proyepoblao6_20/P_200520II_Ajustadosgruposedad.xls

Espinosa, P., Vaca, R., Abad, J. y Crissman, C. (1997). Raíces y tubérculos andinos cultivos marginados en el Ecuador. Situación actual y limitaciones para la producción. Quito: Ediciones Abya-Yala.

Esquema de Ordenamiento Territorial de Turmequé [EOTT] (1999). Turmequé: Alcaldía Municipal.

Follér, M. (2002). Del conocimiento local y científico al conocimiento situado e híbrido. Ejemplos de los shipibo-conibo del este peruano. Revista Anales, (5), 6I-84. Instituto Geográfico Agustín Codazzi [IGAC] (2012). Atlas de la distribución de la propiedad rural en Colombia. Bogotá D. C.: Imprenta Nacional de Colombia Leff, E. (2008). Discursos sustentables. México D. F.: Siglo XXI Editores. León, T. (2010). Agroecología: desafíos de una ciencia ambiental en construcción. En Vertientes del pensamiento agroecológico. Fundamentos y aplicaciones (pp. 45-38). Bogotá D. C.: Opciones gráficas.

Lima, M. y Logroño, J. (2005). Democratización del poder y construcción desde la identidad local desde las mujeres. En Identidad y ciudadanía de las mujeres (pp. 319-420).Quito: Abya-Yala. 
Martínez, M. (2004). Ciencia y arte en la metodología cualitativa. México D. F.: Trillas.

Mora, J. (2008). Persistencia, conocimiento local y estrategias de vida en sociedades campesinas. Revista de Estudios Sociales, (29), I23-133.

Morales, J. (2004). Sociedades rurales y naturaleza. En busca de alternativas hacia la sustentabilidad. Guanajuato: Iteso.

Pengue, W. (2005). Agricultura industrial y transnacionalización en América Latina. ¿La transgénesis de un continente? México D. F.: Pnuma.

Pérez, M. y Clavijo, N. (20I2). Experiencias y enfoques de procesos participativos de innovación en agricultura. El caso de la Corporación PBA en Colombia. Roma: FAO.

Plan de Ordenamiento Territorial del Cantón Colta [POTC] (20II). Colta: Alcaldía del Cantón Colta.

PNUD (20II). Colombia Rural. Razones para la esperanza. Informe nacional de desarrollo bumano. Bogotá D. C.: INDH, Autor.

Proaño, M. (20II). Sistema de Innovación Tecnológica Participativa (Sitpa). En Pontificia Universidad Javeriana (Comp.), VII Seminario Internacional de Desarrollo Rural. Mundos rurales y transformaciones globales: desafios y estrategias de respuesta (pp. 45-52). Bogotá D. C.: Editorial Pontificia Universidad Javeriana.

Ruiz, M. (2009). Las zonas de agrobiodiversidad y el registro de cultivos nativos. Aprendiendo de nosotros mismos. Lima: Lerma Gómez-EIRL.

Tapia, N. (2002). Agroecología y agricultura campesina sostenible en los Andes bolivianos. La Paz: Agruco, Plural.

Tapia, C., Estrella, J., Monteros, A., Valverde, F., Nieto, M. y Córdova, J. (2004). Manejo y conservación de RTAs in situ en fincas de agricultores y ex situ en el Banco de Germoplasma de Iniap. En Raíces y tubérculos andinos. Alternativas para la conservación y uso sostenible en el Ecuador (pp. 3I-74). Quito: Iniap.

Tapia, E. y Rosas, A. (1998). Agrobiodiversidad en La Encañada. Sistematización de las experiencias de conservación in situ de los recursos fitogenéticos. Cajamarca: Condesan, Aspaderuc, CIP, GTZ.

Toledo, V. y Barrera, N. (2008). La memoria biocultural. La importancia ecológica de las sabidurías tradicionales. Barcelona: Icaria.

Woodley, E. (2006). Conocimiento autóctono: un marco conceptual y un caso de las islas Salomón. En J. Gonsalves, T. Becker, A. Braun, D. Campilan, H. Chávez, E. Fajber et al. (Eds.), Investigación y desarrollo participativo para la agricultura y el manejo sostenible de recursos naturales (pp. 76-86). Ottawa: Centro Internacional de la Papa, Centro Internacional para el Desarrollo. 